\title{
Anticoagulation for atrial fibrillation in active cancer (Review)
}

\author{
DIMITRIOS FARMAKIS ${ }^{1}$, PAVLOS PAPAKOTOULAS ${ }^{2}$, ELENI ANGELOPOULOU ${ }^{3}$, THEODOROS BISCHINIOTIS $^{4}$, \\ GEORGE GIANNAKOULAS $^{5}$, PANAGIOTIS KLIRIDIS ${ }^{6}$, DIMITRIOS RICHTER ${ }^{7}$ and IOANNIS PARASKEVAIDIS $^{8}$ \\ ${ }^{1}$ Department of Physiology, University of Cyprus Medical School, Nicosia 2029, Cyprus; \\ ${ }^{2}$ First Department of Clinical Oncology, 'Theagenio' Anticancer Hospital, Thessaloniki 546 39; \\ ${ }^{3}$ Department of Cardiology, 'Agioi Anargyroi' General Oncology Hospital, Athens 145 64; ${ }^{4}$ Department of Cardiology, \\ 'Theagenio' Anticancer Hospital, Thessaloniki 546 39; ${ }^{5}$ Department of Cardiology, AHEPA General Hospital, \\ Aristotle University of Thessaloniki, Thessaloniki 546 21; ${ }^{6}$ Department of Cardiology, 'Agios Savvas' \\ General Anti-Cancer Hospital, Athens 115 22; ${ }^{7}$ Department of Cardiology, Athens Euroclinic, Athens 115 21; \\ ${ }^{8}$ Department of Therapeutics, 'Alexandra' General Hospital, \\ National and Kapodistrian University of Athens Medical School, Athens 115 28, Greece
}

Received November 9, 2021; Accepted February 2, 2022

DOI: $10.3892 / \mathrm{ol} .2022 .13244$

\begin{abstract}
Atrial fibrillation (AF) may often pre-exist in patients with newly diagnosed cancer or occur with increased frequency shortly after cancer diagnosis. Patients with active cancer and AF have a particularly high risk of thromboembolic complications, as both conditions carry a risk of thrombosis. Thromboembolic risk is determined by several factors, including advanced age, sex (females), cancer histology (adenocarcinomas), location (e.g., pancreas, stomach), advanced stage, anticancer regimens (e.g., platinum compounds, anti-angiogenic therapies, immune modulators), comorbidities (e.g., obesity, kidney disease) and concurrent therapies (e.g., surgery, central catheters). Physicians are often reluctant to prescribe anticoagulants to patients with active cancer and AF, mainly due to fear of bleeding complications, which is partly related to the paucity of evidence in the field. Decision making regarding anticoagulation for the prevention of ischemic stroke and systemic embolism in patients with active cancer and AF may be challenging and should not simply rely on the risk prediction scores used in the general AF population. By contrast, the administration and choice of anticoagulants should be based on the comprehensive, individualized and periodic evaluation of thromboembolic and bleeding risk, drug-drug interactions, patient preferences and access to therapies.
\end{abstract}

Correspondence to: Professor Dimitrios Farmakis, Department of Physiology, University of Cyprus Medical School, Shakolas Educational Center for Clinical Medicine, Palaios Dromos Lefkosias Lemesou 215/6, Aglantzia, Nicosia 2029, Cyprus

E-mail: farmakis.dimitrios@ucy.ac.cy; dimitrios_farmakis@yahoo.com

Key words: cancer, atrial fibrillation, anticoagulation, low molecular weight heparins, direct oral anticoagulants

\section{Contents}

1. Introduction

2. Thrombosis in active cancer: An overview

3. Atrial fibrillation in cancer

4. Anticoagulation strategies for atrial fibrillation in cancer

5. Conclusions

\section{Introduction}

Cancer-associated thrombosis (CAT), including venous and arterial thromboembolic events, is a frequent complication in cancer that has a significant impact on patients' morbidity and mortality and often renders their management challenging (1). The risk of CAT is increased in patients with active cancer, in whom the bleeding complications of anticoagulation therapy may also be frequent (2). Cancer is further associated with atrial fibrillation (AF) (3). Some cancer patients, particularly elderly ones, have prevalent $\mathrm{AF}$ at the time of cancer diagnosis, while others will develop AF in the course of the malignancy, partly because of cancer and its therapy. AF carries per se a 5 -fold risk of stroke and systemic thromboembolism (4). Consequently, it has been shown that the coexistence of $\mathrm{AF}$ increases the risk of thromboembolism in patients with cancer (5).

The present report, derived by a meeting of an interdisciplinary panel of experts held in September 2020 in Athens, Greece, addresses the issue of anticoagulation for cancer-associated AF. Focusing on patients with active malignancies, the paper describes the difficulties in decision making that result from the particular features of cancer patients and the relative paucity of evidence and proposes an approach to anticoagulation based on the existing data, where available, the current practice concerning anticoagulation for cancer-associated venous thromboembolism and the limitations of different anticoagulants in the setting of active cancer. 


\section{Thrombosis in active cancer: An overview}

Active cancer. There is not a widely accepted definition of active cancer. The term is generally used to describe patients with recent cancer diagnosis (i.e., within 6 months), those being currently or having been recently treated with anticancer therapies and those with metastatic, locally advanced, recurrent, inoperable or end-stage disease $(6,7)$. Patients with active cancer are more prone to disease-related complications, including a high risk of thromboembolic and bleeding complications $(2,6,8)$. As a result, decision making for anticoagulation treatment and prophylaxis in these patients may be challenging.

Epidemiology. Cancer-associated thrombosis (CAT) is the second-leading cause of death in patients with malignancies, after cancer progression, accounting for $9 \%$ of deaths in a cohort of 4,466 patients (9). In addition to increased morbidity and mortality, CAT further affects ongoing anticancer therapies, escalates patients' psychological burden and distress and increases healthcare costs (10-14).

Cancer-associated venous thromboembolism (VTE), including deep vein thrombosis and pulmonary embolism, represents $30 \%$ of all VTE cases, while cancer increases the age- and sex-adjusted risk of VTE by 5 -fold $(9,15,16)$. VTE, in turn, confers a 4-fold risk of death in patients with cancer (17). Although the term CAT has previously been used to describe VTE, thromboembolic complications in cancer also arise in arterial sites, including myocardial infarction, ischemic stroke and peripheral arterial embolism (18). A new cancer diagnosis carries a 2-fold risk of arterial events. In a large dataset of 279,719 pairs of cancer patients and matched controls, the cumulative incidence of arterial thromboembolism within 6 months from cancer diagnosis was $4.7 \%$ compared to $2.2 \%$ in controls (19).

Pathophysiology. The pathophysiology of CAT is defined by the interaction among three main factors, the thrombogenic effects of cancer, the procoagulant properties of anticancer treatment and patient-related factors. More specifically, cancer cells may directly activate coagulation by the expression of tissue factor (TF) and the release of TF-expressing microparticles and cancer procoagulant factor (20). At the same time, cancer may lead to indirect activation of the coagulation cascade and platelets and inhibition of anticoagulant pathways and fibrinolysis through the induction of a systemic inflammatory reaction (20). The risk of thrombosis is diverse in different cancer types; pancreatic and stomach adenocarcinomas are associated with the highest risk, while haematological malignancies and lung, gynaecological, brain, renal and bladder cancer also confer an increased risk (21). Certain anticancer therapies also bear procoagulant properties resulting from endothelial cell injury or systemic inflammation (21). Anticancer drugs with increased risk of venous or arterial thromboembolism include platinum compounds (cisplatin), anti-angiogenic agents (e.g., bevacizumab, sunitinib, pazopanib), BCR-ABL inhibitors (e.g., nilotinib, ponatinib), immune modulators (e.g., thalidomide, lenalidomide), proteasome inhibitors (e.g., carfilzomib), antimetabolites (e.g., 5-fluorouracil) and hormonal agents (e.g., tamoxifen and aromatase inhibitors) (22). Besides specific anticancer agents, surgery, central venous catheters and supportive therapies such as blood transfusions and erythropoietin-stimulating agents are also associated with increased thromboembolic risk (21). Patient-related risk factors for thromboembolism include female sex, advanced age, obesity, previous history of arterial or venous thromboembolism, comorbidities such as infection, renal or pulmonary disease, prolonged bed rest, poor performance status and hereditary prothrombotic defects (e.g., factor V Leiden) (23). A number of biomarkers have further been associated with an increased risk of CAT, including general haematological or biochemical markers such as white blood cell and platelet counts and C-reactive protein, thrombosis-related markers such as D-dimers and tissue factor (activity or antigen) and adhesion molecules such as P-selectin (23). As previously brought out, AF may often be encountered in patients with active cancer, hence increasing further the risk of stroke and systemic thromboembolic events (3). The risk factors for CAT are summarized in Fig. 1.

\section{Atrial fibrillation in cancer}

Atrial fibrillation $(A F)$. AF is defined as 'a supraventricular tachyarrhythmia with uncoordinated atrial electrical activation and consequently ineffective atrial contraction' (24). The electrocardiographic characteristics of AF include i) irregularly irregular R-R intervals (when atrioventricular conduction is not impaired); ii) absence of distinct repeating $\mathrm{P}$ waves; iii) irregular atrial activations (24). AF is the most common sustained arrhythmia posing a significant burden to patients and healthcare systems worldwide.

Epidemiology. The coexistence of AF and cancer has lately attracted the attention of clinicians treating patients with malignancies $(3,25)$. In a large cohort of 833,520 patients from 26 major healthcare systems in US, a new cancer diagnosis was followed by a 4.4-fold age-adjusted risk of incident AF within the first year (26). The risk ratio fell significantly to 1.22-1.30 beyond the first year of cancer diagnosis, indicating a stronger association of $\mathrm{AF}$ with active cancer. A particularly common form of AF in patients with cancer is peri-operative AF (3). In a cohort of 13,906 patients undergoing pulmonary resection for lung cancer, perioperative AF occurred in $12.6 \%$ of patients (27). Peri-operative AF seems to occur more frequently in patients with advanced age and cancer stage and coexistence of cardiovascular comorbidities and in association with prolonged operation and extensive tissue resection (3).

It has further been suggested that there is a reciprocal relationship between AF and cancer. This is supported by evidence showing an increased incidence of cancer diagnosis in patients with prevalent $\mathrm{AF}$ of recent onset, indicating that AF might be a potential marker of occult cancer. In a large cohort of 269,742 individuals, there was a 5-fold standardized risk for cancer within the first 3 months of AF diagnosis (28) further confirmed by other studies $(26,29,30)$. Although causality cannot be supported by such epidemiological data, this reverse relationship stresses at least the common risk factors that the two entities share, such as ageing, obesity or smoking, on a background of a systemic low-level inflammation (31). A recent systematic review and meta-analysis showed 


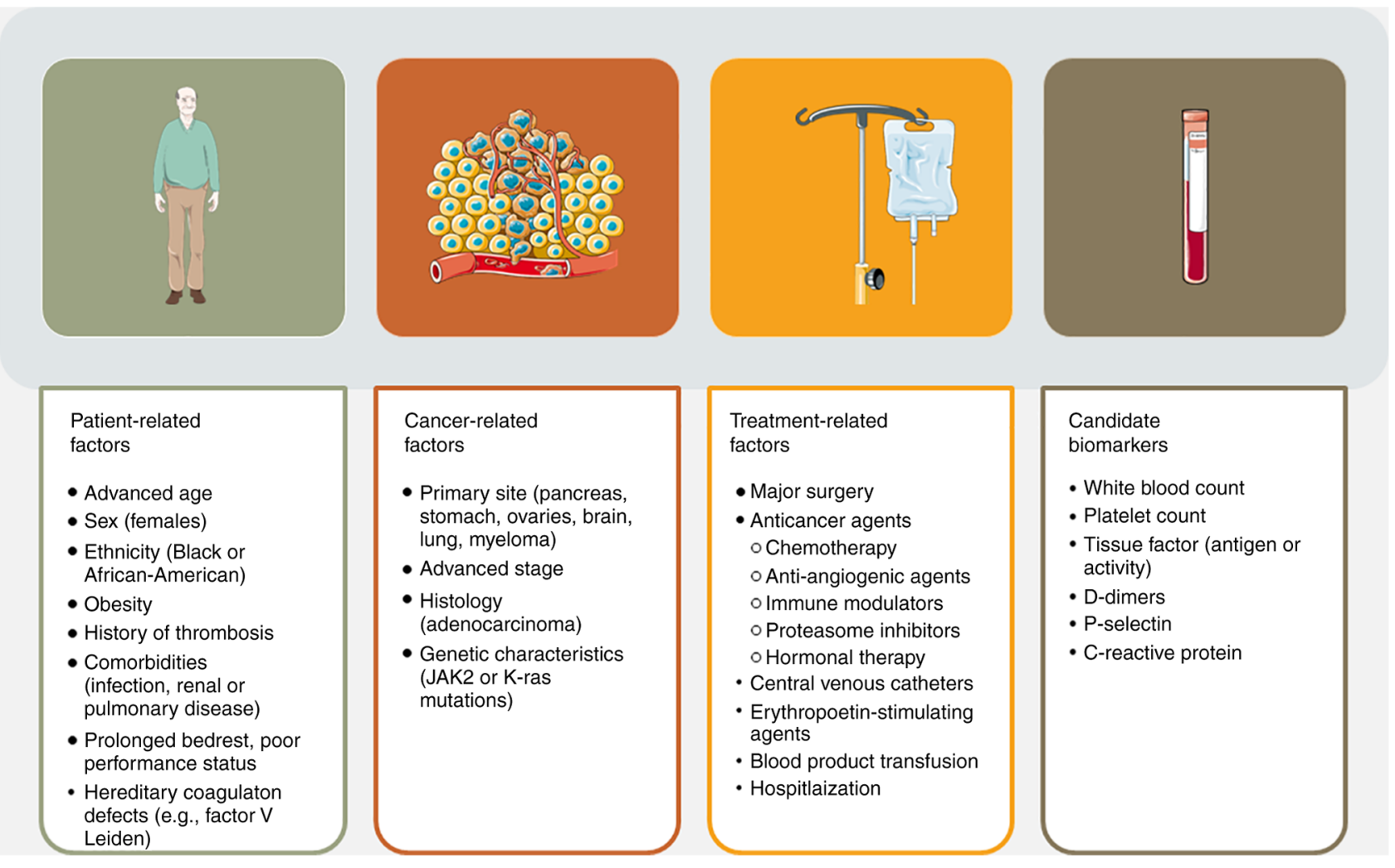

Figure 1. Risk factors for thrombosis in patients with cancer (content modified from ref. 23).

that bleeding under a direct oral anticoagulants (DOAC), was associated with 6-fold risk of cancer detection, while bleeding under a vitamin $\mathrm{K}$ antagonist (VKA) with a 15-fold risk (32). Similarly to non-cancer patients, prevalent or new-onset AF increases the risk of thromboembolic events in patients with a malignancy. In a retrospective cohort of 24,125 patients with newly diagnosed malignant disease, $2.4 \%$ of patients had prevalent $\mathrm{AF}$ at the time of cancer diagnosis, while another $1.8 \%$ developed AF after cancer diagnosis; both baseline and new-onset AF were associated with a significantly higher incidence of thromboembolism compared to the absence of AF, even after adjustment for age, sex and comorbidities (5).

Pathophysiology. Several mechanisms have been proposed for the pathogenesis of AF in patients with malignancies (33). Cancer may induce AF directly through the invasion of the heart by primary or metastatic cardiac tumours or tumours of adjacent or remote organs. More commonly, cancer may indirectly cause AF through a series of potential mechanisms such as fluid imbalance, hypoxia, electrolyte and metabolic abnormalities, infection, anaemia, autonomic nervous system dysregulation and paraneoplastic manifestations.

Anticancer drugs and other supportive therapies have further been associated with AF. An analysis of the World Health Organization's pharmacovigilance database VigiBase identified a long list of systemic anticancer therapies associated with AF including alkylating agents (e.g., cisplatin, dacarbazine), anthracyclines (e.g., doxorubicin, idarubicin, daunorubicin), antimetabolites (e.g., gemcitabine, clofarabine), taxanes (e.g., docetaxel), bruton kinase inhibitors (e.g., ibrutinib), BCR-Abl inhibitors (e.g., nilotinib, ponatinib), proteasome inhibitors (e.g., bortezomib), immune checkpoint inhibitors (e.g., ipilimumab), immunomodulatory agents (e.g., aldesleukin, pomalidomide, lenalidomide), monoclonal antibodies (e.g., rituximab) and androgen deprivation agents (e.g., abiraterone) (34,35). Anticancer drug-induced AF may manifest during or shorty (within $24 \mathrm{~h}$ ) after drug administration, as in the case of cisplatin or gemcitabine, or develop several days or even months later, as, for example, with ibrutinib (36).

In addition, surgery, particularly pulmonary resection or other extensive operations are often followed by peri-operative AF (3).

Besides cancer and anticancer therapies, more importantly, as previously implied, cancer and AF share common risk factors that could pave the way simultaneously to the two conditions.

As in the case of VTE, AF-associated thrombosis can be explained by the Virchow's triad. AF is associated with stasis due to stagnant blood flow in the atria, wall changes due to atrial remodelling and endothelial injury, and hypercoagulability due to the activation of platelets and coagulation factors and inflammation $(33,37)$. The interaction among cancer, anticancer therapy and AF is outlined in Fig. 2.

Inflammation seems to be a common denominator underlying cancer, AF and thrombosis. It seems to play an important role in tumour survival, proliferation, angiogenesis and metastasis (38). As previously stated, inflammation is believed to be involved in CAT $(39,40)$, while there is evidence for a pathogenic role of blood coagulation in tumour growth and metastasis $(41,42)$. An intrinsic pathway of inflammation (driven in tumour cells), as well as an extrinsic pathway 


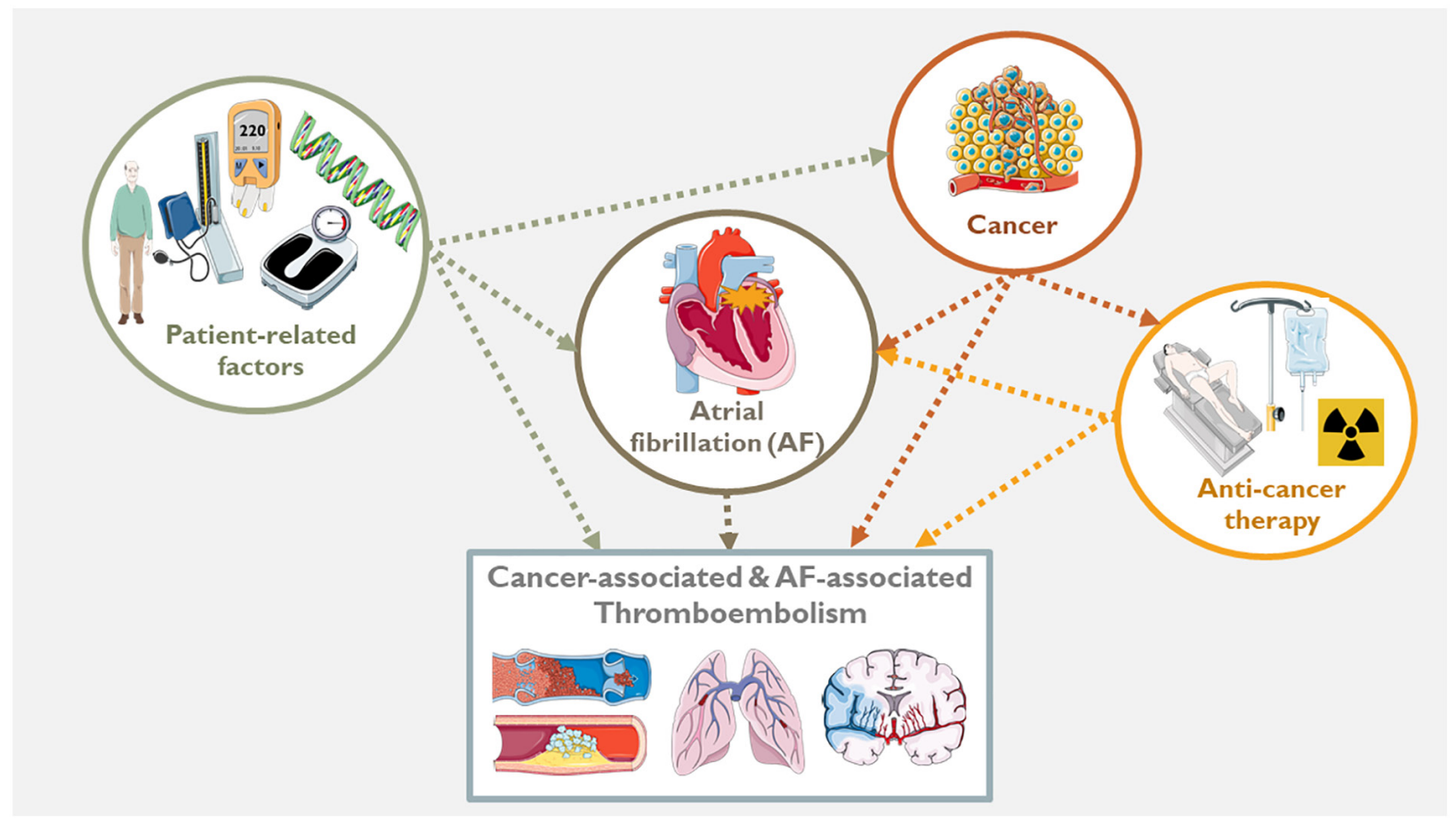

Figure 2. The complex interplay among cancer, anticancer therapy and AF. Cancer and its therapy may lead to AF. At the same time, cancer and AF share common risk factors, including aging, cardiometabolic comorbidities such as hypertension, diabetes mellitus and obesity, and genetic predisposition. All the above, cancer, anticancer therapy, $\mathrm{AF}$ and their common patient-related risk factors, are predisposing factors for thromboembolic complications including stroke. AF, atrial fibrillation.

(in tumour-infiltrating leukocytes) both seem to contribute to tumour progression (43). Inflammation, activated by cardiometabolic risk factors and comorbidities is further believed to hold a key role in the pathogenesis of atrial disease, a constellation of structural, electrical and functional atrial changes that underlies the development of AF (44). The production of reactive oxygen species (ROS), which are by-products of cellular metabolism and oxygen use and have been associated with an increased risk of cancer development via DNA damage and genetic destabilization $(43,44)$, seems to be an important player in the association between inflammation on one hand and AF and cancer on the other (45). An increase in inflammatory markers such as C-reactive protein, tumour necrosis factor-a and interleukins 2, 6, and 8 has actually been found in patients with AF (3). Additionally, inflammation can be both a cause as well as a consequence of VTE. VTE-induced inflammation leads to the impaired thrombus recovery and the increased risk of VTE-related complications (46). VTE and AF share many common risk factors, including old age, obesity, heart failure, and inflammatory states. Moreover, VTE and more specifically pulmonary embolism (PE) may lead to AF through right-sided pressure overload. Epidemiological studies indicate that AF can be seen as a presenting sign, during the early phase of PE, or develop later in the course of recovery from PE (47).

\section{Anticoagulation strategies for atrial fibrillation in cancer}

The management of AF in patients with malignancies in terms of rhythm and rate control follows the strategies that apply to the general AF population, taking under consideration cancer prognosis and the potential interactions of cardioactive medications with anticancer agents and supportive therapies (4).

Challenges. There are important challenges in decision making regarding anticoagulation therapy for stroke and systemic embolism prevention in AF patients with malignancies. Patients with AF and active cancer may have a higher thrombotic risk compared to those with AF due to specific cancer histology and location and specific anticancer therapies, as previously noticed. On the other hand, patients with active cancer may also have a higher risk of bleeding, also associated with cancer or anticancer therapies $(2,8,48)$. Patients with increased risk of bleeding include those with intracranial tumours, gastrointestinal or genitourinary cancer or haematological malignancies, and those having thrombocytopenia either due to bone marrow invasion or due to myelotoxicity from systemic anticancer therapy or irradiation. In a prospective cohort study on 2,288 patients with AF treated with DOAC, the risk of both thromboembolic events and major bleeding was 4-fold higher in patients with active cancer compared to those without cancer or those with non-active cancer [adjusted hazard ration (HR) of thromboembolism, 4.03 (1.35-12.03); adjusted HR for major bleeding, 3.87, 95\% CI, 2.16-6.94)].

It has been previously highlighted that patients with prevalent AF may have an increased probability of being diagnosed with cancer, particularly during the first months of AF diagnosis. Furthermore, because of advancing age and accumulation of other comorbid conditions, the incidence of cancer is steadily increasing with time after AF diagnosis. In a Danish population cohort of 55,100 individuals, up to one fourth of individuals who developed AF were subsequently diagnosed 
with cancer over a 12-year period following AF diagnosis (29). In these patients, the decision to continue or modify their previous anticoagulation regimen before the initiation of anticancer therapy and during the active phase of cancer may pose an additional challenge.

Scores that are widely recommended and used for the prediction of thromboembolic or haemorrhagic risk in the general AF population have not been sufficiently validated in patients with cancer, including the $\mathrm{CHA}_{2} \mathrm{DS}_{2} \mathrm{VASc}$ score (Congestive heart failure, Hypertension, Age $\geq 75$ years, Diabetes mellitus, prior Stroke or transient ischemic attack or thromboembolism, Vascular disease, Age 65-74, Sex category). Similarly, the HAS-BLED score (Hypertension, Abnormal renal or liver function, Stroke, Bleeding history or predisposition, Labile INR, Elderly, Drugs or alcohol), used for the estimation of bleeding risk in the general AF population, seems to underestimate this risk in patients with $\mathrm{AF}$ and concomitant cancer, according to a large cohort study (49).

At the same time, anticoagulants may interact with anticancer medications and other supportive therapies prescribed in patients with cancer that may either attenuate or intensify the anticoagulant effect, thus increasing the risk of thromboembolic or bleeding complications, respectively (50). Drug-drug interactions (DDI) are a growing concern in patients with cancer (51). It has been reported that at least $46 \%$ of cancer patients were exposed to at least one DDI (52); $84 \%$ of these DDIs were associated with a deterioration of patients' status and required treatment while $14 \%$ were even life-threatening or exposed patients to permanent damage. The risk of DDI is even more pronounced in elderly patients (53). On the other hand, among 115,362 patients with AF or VTE who were newly prescribed DOACs, one third of patients presented one potential DDI and $12.6 \%$ had at least 2 DDIs (54); patients with bleeding had an $85 \%$ higher occurrence of DDIs when compared to those without. It appears that a regular assessment of potential DDI should be implemented, and therapies need to be adequately adjusted.

Finally, cancer patients are often elderly and fragile individuals, suffering from additional conditions and receiving additional medications. Frailty, comorbidities and polypharmacy may impair drug tolerance and safety and complicate DDI. As previously spotlighted, AF may be prevalent at the time of cancer diagnosis and these patients may have already been prescribed a certain anticoagulation regimen that might not be appropriate for a given cancer type or anticancer therapy plan (29).

In this context, oncology clinicians need to monitor the anticoagulant effect and make dose adjustments. Though, clinical studies are conducted with a fixed dose of DOACs and do not assess clinical outcomes based on DDI or coagulation assays. Therefore, no evidence based recommendation for drug concentration measurements, coagulation tests, assay standardization, or target therapeutic ranges has been clearly established for DOAC (55).

According to an international questionnaire-based survey addressing the concerns and prescribing preferences of 960 cardiologists regarding AF in cancer, the most important limitations in the prescription of anticoagulants for stroke and systemic embolism prevention included the lack of dedicated clinical trials (34\%), DDI with anticancer agents (32\%) and the need to monitor the anticoagulant effect and make dose adjustments (19\%) (56). In accordance to these findings, there seems to be a gap in the treatment of AF in cancer patients, with low usage of thromboembolic therapy that is not prescribed in $44 \%$ of patients despite a high thromboembolic risk and an acceptable bleeding risk (57).

\section{Advantages and disadvantages of available anticoagulants}

Vitamin K antagonists. Vitamin K antagonists [VKA] bear many disadvantages in the setting of cancer. They have multiple interactions with numerous anticancer agents (58) and a narrow therapeutic window with a low likelihood to achieve optimal TTR due to gastrointestinal complication such as vomiting, malnutrition and hepatic dysfunction (59). In a study of patients with prevalent AF and newly diagnosed cancer, there was no benefit from VKA therapy mainly due to suboptimal INR control, as only $12 \%$ of patients were in optimal INR range (60). VKA has also been associated with a 6-fold higher risk of bleeding in patients with cancer compared to those without (61). These drugs are also difficult to handle peri-operatively. However, VKA remain the only anticoagulants currently indicated for valvular AF, including patients with moderate or severe mitral valve stenosis and those with mechanical valve prosthesis (4).

Low molecular weight heparins. Low molecular weight heparins [LMWH] have long been the preferred agents for the primary and secondary prevention of VTE in patients with cancer and there is considerable accumulated experience with their use in this setting $(62,63)$. LMWH further lack notable interactions with anticancer drugs and they are administered parenterally and therefore their absorption is not affected by gastrointestinal complications such as vomiting. It has also been suggested that LMWH may bear anti-tumour properties, including anti-proliferative, anti-angiogenic and anti-metastatic actions along with favourable effects on cellular adhesion, epithelial-mesenchymal transition [EMT], extracellular matrix heparinase and metalloproteinases, cancer-drug resistance and tumour micro-environment (64-66). The potential anti-inflammatory effects of LMWH may also be relevant, given the key pathogenetic role of inflammation in cancer, $\mathrm{AF}$ and thrombosis (67). These additional properties may be related to the survival advantage associated with LMWH in cancer patients without thromboembolic events in a small clinical study (68).

There is no clear evidence on the effectiveness of LMWH in stroke or systemic embolism prevention in AF, although these drugs are often used as alternatives to oral anticoagulants in $\mathrm{AF}$ patients in different settings including peri-procedural bridging and transoesophageal echocardiography-guided cardioversion (69-71). The parenteral route of administration may impair patients' compliance, although evidence suggests that LMWH are acceptable by patients in the context of cancer (72).

Direct oral anticoagulants. Direct oral anticoagulants [DOAC] are currently indicated as first-line agents for stroke or systemic embolism prevention in the general AF population (4). DOAC have a lower risk of intracranial bleeding compared to VKA, while there is also the possibility of a reversal agent, currently for dabigatran and soon for the rest of DOAC. In patients with 
cancer, recent evidence from randomized controlled trials have shown that DOAC are viable alternatives to LMWH for VTE with a higher efficacy in preventing VTE recurrence but with worse safety in terms of bleeding complications (73-77).

Concerning AF in cancer, evidence derived by secondary analyses of randomized trials or observational studies shows that DOAC, and more specifically rivaroxaban, apixaban and edoxaban, seem to have preserved efficacy and safety over VKA for stroke and systemic embolism prevention in patients with AF and cancer (78-82). In addition, two meta-analyses including the above secondary analyses along with observational retrospective studies have further advocated for better outcomes in terms of thromboembolic and bleeding risks with DOAC vs. VKA in patients with AF and cancer $(83,84)$. However, patients with cancer, particularly those with an active malignancy, were considerably underrepresented in these trials. In ROCKET-AF that assessed the efficacy and safety of rivaroxaban vs. warfarin in AF, any history of cancer was present in $4.5 \%$ of patients, while metastatic cancer was present in less than $0.1 \%$ of cases (78). Similarly, in ARISTOTLE on apixaban, $6.6 \%$ of patients enrolled had a history of cancer, while only $0.7 \%$ had an active malignancy (79). Finally, in ENGAGE-AF on edoxaban, patients with cancer were generally excluded, yet a $5.5 \%$ of the study population developed active cancer in the course of the trial after a variable time period from study onset (80). Furthermore, cancer populations across studies included in the meta-analyses were heterogeneous, which might have led to uncontrolled confounding.

All four licensed DOAC are substrates for P-glycoprotein and therefore should be avoided with drugs that are potent inhibitors or inducers of P-glycoprotein (50). In addition, rivaroxaban and apixaban are also metabolized by cytochrome P450 (CYP3A4) and should be used with extreme caution with other inducers or inhibitors of CYP3A4 (85). DOAC may therefore have significant interactions with anticancer agents, other supportive therapies prescribed in patients with cancer, but also food, herbs and over-the-counter [OTC] drugs. In a recent report, $33 \%$ of patients receiving apixaban had at least one OTC product with potentially serious apixaban interactions daily or most of the days (86). The different DOAC have variable degrees of renal clearance and their activity can be affected in patients with cancer and chronic kidney disease or worsening renal function (44). Due to their oral route of administration, DOAC have an unpredictable absorption in the case of gastrointestinal complications such as vomiting.

Current practice and recommendations. The available guidelines, position statements or other documents on anticoagulation for AF in cancer recommend the use of general scores such as $\mathrm{CHA}_{2} \mathrm{DS}_{2}$ VASc and HAS-BLED (hypertension, abnormal renal or liver function, stroke, bleeding, labile international normalized ratio, elderly, drugs or alcohol) $(3,87,88)$. In addition to these scores, it is, however, reinforced that supplementary parameters should be taken under consideration, mainly for safety reasons, such as platelet count or tumour location $(3,87,88)$.

Patients with cancer are often elderly with multiple comorbidities and therefore are classified as individuals with increased risk of thromboembolism by the general prediction scores. In a recent retrospective analysis on 472 cancer patients with AF or atrial flutter, the mean $\mathrm{CHA}_{2} \mathrm{DS}_{2}$-VASc was 2.8 (89); 44\% did not receive anticoagulation, despite the fact that only $18 \%$ had platelet counts $<50,000 / \mathrm{ml}(89)$. In practice, anticoagulation seems to be under-prescribed in cancer patients with AF, including those who are hospitalized or who are actively treated with anticancer treatment. In a study depicting anticoagulation patterns in 2015 among 394 hospitalized patients with active cancer, AF and a mean $\mathrm{CHA}_{2} \mathrm{DS}_{2}$-VASc of $2.2,65 \%$ of patients did not receive any anticoagulation at all $(25 \%)$ or they were treated with prophylactic-dose LMWH (35\%), while only 39\% were treated with therapeutic-dose LMWH and $1 \%$ with a DOAC (90).

A proposed approach to anticoagulation in active cancer. Given the increased thromboembolic and bleeding risk related to active cancer and the lack of solid evidence on the proper anticoagulation regimen for AF in these patients, the decision on the onset and the choice of anticoagulants should be based on the comprehensive and individualized assessment of four main factors: i) the risk of thrombosis associated with both cancer and AF, ii) the risk of bleeding, iii) the interactions of anticoagulants with anticancer and other therapies and iv) the patient's informed preferences and access to anticoagulants. It has recently been proposed that these four domains are summarized by the acronym TBIP (T, 'thrombosis'; B, 'bleeding'; I, 'interactions'; P, 'patient'; Fig. 3).

Anticoagulation should not be prescribed, at least temporarily, in patient with very high risk of bleeding, as in the case of active haemorrhage, severe thrombocytopenia $\left(<25,000 \times 10^{9} / 1\right)$ or recent or evolving intracranial lesions. In these patients, when the risk of thromboembolism is significant and the life expectancy reasonable, left atrial appendage occlusion may be an option (4).

VKA remain the only indicated anticoagulants for patients with valvular AF. LMWH, at standard or adjusted therapeutic dose, should be preferred in patients with increased risk of bleeding, such as those with active gastrointestinal or genitourinary cancer or gastrointestinal mucosal abnormalities, platelet counts of $25,000-50,000 \times 10^{9} / 1$, as well as for those experiencing significant gastrointestinal toxicity or severe renal dysfunction. Regarding renal function, in patients with an estimated glomerular filtration rate (eGFR) $<15 \mathrm{ml} / \mathrm{min} / 1.73 \mathrm{~m}^{2}$, all DOAC are contraindicated, while in those with an eGFR of $15-30 \mathrm{ml} / \mathrm{min} / 1.73 \mathrm{~m}^{2}$, dabigatran is contraindicated and the rest of DOAC should administered in halved dose (rivaroxaban and edoxaban dose should also be halved for an eGFR of $30-50 \mathrm{ml} / \mathrm{min} / 1.73 \mathrm{~m}^{2}$ ) (44).

LMWH should also be preferred in patients receiving anticancer agents or other therapies with significant interactions with oral anticoagulants (potent inhibitors or inducers of P-glycoprotein and/or CYP3A4). Among common anticancer agents, doxorubicin, vinblastine, sunitinib, vandetanib and dexamethasone are strong inducers of P-glycoprotein, while imatinib is a strong inhibitor of P-glycoprotein and all DOACs should be avoided in conjunction with these agents (85). In addition, paclitaxel is a moderate inducer of CYP3A4 and imatinib a moderate inhibitor of CYP3A4 and rivaroxaban and apixaban should be used with extreme caution with these drugs (85). In any case, the potential drug-drug interactions should be checked before prescription of a DOAC in a patient with active cancer. 


\begin{tabular}{|l|}
\hline Assessment of patients with $\mathrm{AF}$ and active cancer \\
\hline Assess Thromboembolic risk: \\
- AF-related risk (eg, $\mathrm{CHA}_{2} \mathrm{DS}_{2}$-VASc score) \\
- Cancer-associated risk (eg, Khorana score) \\
Assess Bleeding risk: \\
- Thrombocytopenia (PTL $<50,000 / \mu \mathrm{l})$ \\
- Gl/GU cancer, Gl comorbidities or toxicity \\
- Recent or evolving intracranial lesions \\
- Recent major bleeding \\
- Severe renal dysfunction (eGFR $\left.<30 \mathrm{ml} / \mathrm{min} / 1.73 \mathrm{~m}{ }^{2}\right)$ \\
- Bleeding risk scores (HAS-BLED, HEMORR ${ }_{2} \mathrm{HAGES}$, other?) \\
Assess drug-drug Interactions (P-glycoprotein, CYP3A4): \\
- Anticancer agents \\
- Supportive therapies \\
Assess Patient access and preferences \\
- Access to drugs, drug availability \\
- Patient preference
\end{tabular}

\begin{tabular}{|c|}
\hline Anticoagulation in specific profiles \\
\hline \multicolumn{1}{|c|}{ Valvular AF } \\
\hline VKA \\
\hline $\begin{array}{c}\text { Low thromboembolic risk or } \\
\text { recent/evolving intracranial lesions or } \\
\text { recent major bleeding }\end{array}$ \\
\hline No anticoagulation \\
\hline $\begin{array}{c}\text { PTL }<50,000 / \mu l \text { or GI/GU cancer or GI } \\
\text { comorbidities or GI toxicity or severe } \\
\text { renal dysfunction or drug-drug } \\
\text { interactions }\end{array}$ \\
\hline Yes \\
\hline \begin{tabular}{l} 
LMWH $\quad$ No \\
\hline
\end{tabular}
\end{tabular}

Figure 3. A proposed approach to anticoagulation for AF in patients with active cancer (Farmakis D: Anticoagulation for atrial fibrillation in active cancer: What the cardiologists think. Eur J Prev Cardiol, 2020, 28: 608-610 (reference 1) by permission of Oxford University Press). AF, atrial fibrillation.

For the rest of patients, DOAC or LMWH may be selected based on the overall thrombotic burden, the local experience or protocols and the preferences and access of patients. In addition, it is crucial that the initial anticoagulation regimen should be revaluated periodically (e.g., every 3 months or more frequently in unstable patients) and updated in accordance to the course of cancer, any modifications in anticancer regimen or changes in patient parameters (e.g., platelet count, renal function, gastrointestinal complications). Such a comprehensive approach can be significantly facilitated if carried out in the context of a well-structured cardio-oncology service and with the close collaboration among all implicated physicians $(91,92)$.

In patients with pre-existing $\mathrm{AF}$ on anticoagulant therapy prior to cancer diagnosis, the anticoagulation regimen should be revisited upon the onset of anticancer therapy. First, oral anticoagulants should be properly discontinued or bridged with LMWH during the perioperative period in the case of cancer surgery. Detailed guidance on perioperative interruption and bridging of oral anticoagulants according to the type of anticoagulant, the type of surgery and other parameters, such as renal function, is provided by dedicated documents (93). Second, the continuation or modification of anticoagulation regimen that the patient received for AF before cancer diagnosis, during systemic anticancer therapy and as long as cancer is in active phase, should be decided based on the previously described approach (Fig. 3). Accordingly, oral anticoagulants (i.e., DOAC or VKA) may need to be replaced by LMWH, at least temporarily, in active cancer patients with significant drug interactions between oral anticoagulants and candidate anticancer drugs, increased bleeding risk (e.g., gastrointestinal tumours, thrombocytopenia) or significant gastrointestinal toxicity (e.g., vomiting) and in accordance with eGFR in the case of worsening renal function. As previously discussed, the anticoagulation regimen should be periodically revisited during the active phase of cancer.

\section{Conclusions}

Advances in cancer screening, diagnosis and mainly treatment allow for a continuously growing population of cancer survivors, in whom the impact of heart disease on morbidity and mortality increases with age $(94,95)$. As AF is an age-related disease, an increasing number of cancer survivors are expected to develop AF (96). In addition, AF, either pre-existing or occurring shortly after cancer diagnosis, may increase the morbidity and mortality of patients with an active malignancy. Despite the considerable improvements in adherence to guideline recommendations regarding anticoagulation in the general AF population (97), this does not seem to be the case in patients with active cancer and AF. This gap in care is partly related to the lack of dedicated randomized trials that are warranted in this particular field. The overall thromboembolic risk in patients with active cancer and AF should not be addressed in the same manner as in patients with AF without cancer, as it combines both AF-associated and cancer-associated risks. Specifically, four factors should be taken under consideration summarized by the acronym TBIP (T, 'thrombosis'; B, 'bleeding'; I, 'interactions'; P, 'patient'). For valvular AF, VKA are the only indicated anticoagulants. For patients with very low thrombotic risk or recent/evolving intracranial lesions or major bleeding, anticoagulation may be deferred. For cases with thrombocytopenia $(<50.000 / \mu 1)$, active gastrointestinal or genitourinary cancer, gastrointestinal comorbidities or toxicity, severe renal dysfunction or drug-drug interactions, LMWH are preferred over DOAC. If there are none of the 
above conditions or limitations, either DOAC or LMWH can be used. Until more solid evidence is available, the comprehensive and periodic assessment of each patient with cancer and AF will allow a tailored approach to anticoagulation, aiming at precision medicine that represents an essential goal in the new but rapidly evolving field of cardio-oncology (91).

\section{Acknowledgements}

Not applicable.

\section{Funding}

No funding was received.

\section{Availability of data and materials}

Not applicable.

\section{Authors' contributions}

DF: conception and design, data collection and interpretation, drafting and final approval of the manuscript. PP: conception, design, critical review and final approval of the manuscript. EA: data collection and interpretation, critical review and final approval of the manuscript. TB: data collection and interpretation, critical review and final approval of the manuscript. GG: conception, design, critical review and final approval of the manuscript. PK: data collection and interpretation, critical review and final approval of the manuscript. DR: data collection and interpretation, critical review and final approval of the manuscript. IP: conception, design, critical review and final approval of the manuscript. Data authentication is not applicable. All authors read and approved the final manuscript.

\section{Ethics approval and consent to participate}

Not applicable.

\section{Patient consent for publication}

Not applicable.

\section{Competing interests}

DF has received lecture honoraria and/or advisory board fees from Abbott Laboratories, Bayer, Boehringer Ingelheim, Leo, Novartis and Orion Pharma. GG has received lecture fees and/or research support from Bayer, Boehringer Ingelheim, Pfizer and Leo Pharmaceutical Hellas. DR has received travel grants, lecture and advisory board fees from Amgen, Sanofi, Bayer, Boehringer, MSD, Leo, Teva, Mylan, Menarini, Unipharma, Servier, AstraZeneca, Vianex and Elpen. The rest of the authors report no conflict of interest.

\section{References}

1. Farmakis D: Anticoagulation for atrial fibrillation in active cancer: What the cardiologists think. Eur J Prev Cardiol 28: 608-610, 2020.
2. Vedovati MC, Giustozzi M, Verdecchia P, Pierpaoli L, Conti S, Verso M, Di Filippo F, Marchesini E, Bogliari G, Agnelli G and Becattini C: Patients with cancer and atrial fibrillation treated with doacs: A prospective cohort study. Int J Cardiol 269: 152-157, 2018.

3. Farmakis D, Parissis J and Filippatos G: Insights into onco-cardiology: Atrial fibrillation in cancer. J Am Coll Cardiol 63: 945-953, 2014.

4. Hindricks G, Potpara T, Dagres N, Arbelo E, Bax JJ, Blomström-Lundqvist C, Boriani G, Castella M, Dan GA, Dilaveris PE, et al: 2020 ESC Guidelines for the diagnosis and management of atrial fibrillation developed in collaboration with the European Association of Cardio-Thoracic Surgery (EACTS): The Task Force for the diagnosis and management of atrial fibrillation of the European Society of Cardiology (ESC) Developed with the special contribution of the European Heart Rhythm Association (EHRA) of the ESC. Eur Heart J 42: 373-498, 2021.

5. Hu YF, Chen TC, Chau GY, Yang TL, Liu CJ, Chen MH, Chang PM, Chen TJ, Hsiao M, Huang CY and Chen SA: Baseline hypertension: New insight into the potential predictors of survival in patients with hepatocellular carcinoma. Int J Cardiol 168: 2979-2981, 2013

6. NICE: Venous thromboembolism in adults. Quality standard [QS201]. https://www.nice.org.uk/guidance/qs201/resources/ venous-thromboembolism-in-adults-pdf-75547409387461. Accessed February 17, 2022.

7. Khorana AA, Noble S, Lee AYY, Soff G, Meyer G, O'Connell C and Carrier M: Role of direct oral anticoagulants in the treatment of cancer-associated venous thromboembolism: Guidance from the SSC of the ISTH. J Thromb Haemost 16: 1891-1894, 2018.

8. Sato T, Aizawa Y, Fuse K, Fujita S, Ikeda Y, Kitazawa H, Takahashi $\mathbf{M}$ and Okabe M: The impact of cancer on major bleeding and stroke/systemic emboli in patients using direct oral anticoagulants: From the database of a single-center registry. J Atrial Fibrillation 11: 2105, 2018.

9. Khorana AA, Francis CW, Culakova E, Kuderer NM and Lyman GH: Thromboembolism is a leading cause of death in cancer patients receiving outpatient chemotherapy. J Thromb Haemost 5: 632-634, 2007.

10. Khorana AA: Venous thromboembolism and prognosis in cancer. Thrombosis Res 125: 490-493, 2010.

11. Elting LS, Escalante CP, Cooksley C, Avritscher EB, Kurtin D, Hamblin L, Khosla SG and Rivera E: Outcomes and cost of deep venous thrombosis among patients with cancer. Arch Intern Med 164: 1653-1661, 2004.

12. Sheard L, Prout H, Dowding D, Noble S, Watt I, Maraveyas A and Johnson M: Barriers to the diagnosis and treatment of venous thromboembolism in advanced cancer patients: A qualitative study. Palliat Med 27: 339-348, 2013.

13. Seaman S, Nelson A and Noble S: Cancer-associated thrombosis, low-molecular-weight heparin, and the patient experience: A qualitative study. Patient Prefer Adherence 8: 453-461, 2014.

14. Khorana AA, Dalal MR, Lin J and Connolly GC: Health care costs associated with venous thromboembolism in selected high-risk ambulatory patients with solid tumors undergoing chemotherapy in the United States. Clinicoecon Outcomes Res 5: 101-108, 2013.

15. Puurunen MK, Gona PN, Larson MG, Murabito JM, Magnani JW and O'Donnell CJ: Epidemiology of venous thromboembolism in the Framingham Heart Study. Thrombosis Res 145: 27-33, 2016.

16. Walker AJ, Card TR, West J, Crooks C and Grainge MJ: Incidence of venous thromboembolism in patients with cancer-a cohort study using linked United Kingdom databases. Eur J Cancer 49: 1404-1413, 2013.

17. Chew HK, Wun T, Harvey D, Zhou H and White RH: Incidence of venous thromboembolism and its effect on survival among patients with common cancers. Arch Intern Med 166: 458-464, 2006.

18. Abdol Razak NB, Jones G, Bhandari M, Berndt MC and Metharom P: Cancer-associated thrombosis: An overview of mechanisms, risk factors, and treatment. Cancers (Basel) 10: 380, 2018.

19. Navi BB, Reiner AS, Kamel H, Iadecola C, Okin PM, Elkind MSV, Panageas KS and DeAngelis LM: Risk of arterial thromboembolism in patients with cancer. J Am Coll Cardiol 70: 926-938, 2017.

20. Donnellan E and Khorana AA: Cancer and venous thromboembolic disease: A review. Oncologist 22: 199-207, 2017.

21. Ay C, Pabinger I and Cohen AT: Cancer-associated venous thromboembolism: Burden, mechanisms, and management. Thromb Haemost 117: 219-230, 2017. 
22. Mosarla RC, Vaduganathan M, Qamar A, Moslehi J, Piazza G and Giugliano RP: Anticoagulation strategies in patients with cancer: JACC review topic of the week. J Am Coll Cardiol 73 $1336-1349,2019$.

23. Khorana AA and Connolly GC: Assessing risk of venous thromboembolism in the patient with cancer. J Clin Oncol 27 4839-4847, 2009.

24. January CT, Wann LS, Calkins H, Chen LY, Cigarroa JE, Cleveland JC Jr, Ellinor PT, Ezekowitz MD, Field ME, Furie KL, et al: 2019 AHA/ACC/HRS Focused Update of the 2014 AHA/ACC/HRS Guideline for the management of patients with atrial fibrillation: A report of the American College of Cardiology/American Heart Association Task Force on Clinical Practice Guidelines and the Heart Rhythm Society. J Am Coll Cardiol 74: 104-132, 2019.

25. Farmakis D and Filippatos G: Arrhythmias in cancer: Rhythm is gonna get you! Eur J Heart Fail 23: 154-156, 2021.

26. Kim LK, Swaminathan RV, Looser P, Minutello RM, Wong SC, Bergman G, Naidu SS, Gade CL, Charitakis K, Singh HS and Feldman DN: Hospital volume outcomes after septal myectomy and alcohol septal ablation for treatment of obstructive hypertrophic cardiomyopathy: US Nationwide Inpatient Database, 2003-2011. JAMA Cardiol 1: 324-332, 2016.

27. Onaitis M, D'Amico T, Zhao Y, O'Brien S and Harpole D: Risk factors for atrial fibrillation after lung cancer surgery: Analysis of the Society of Thoracic Surgeons general thoracic surgery database. Ann Thorac Surg 90: 368-374, 2010.

28. Ostenfeld EB, Erichsen R, Pedersen L, Farkas DK, Weiss NS and Sorensen HT: Atrial fibrillation as a marker of occult cancer. PLoS One 9: e102861, 2014.

29. Vinter N, Christesen AMS, Fenger-Gron M, Tjonneland A and Frost L: Atrial fibrillation and risk of cancer: A danish population-based cohort study. J Am Heart Assoc 7: e009543, 2018 .

30. Conen D, Wong JA, Sandhu RK, Cook NR, Lee IM, Buring JE and Albert CM: Risk of malignant cancer among women with new-onset atrial fibrillation. JAMA Cardiol 1: 389-396, 2016.

31. Farmakis D, Parissis J, Karavidas A, Karvounis C, Triposkiadis F, Filippatos G and Lekakis J; Collaborators: In-hospital management of acute heart failure: Practical recommendations and future perspectives. Int J Cardiol 201: 231-236, 2015.

32. Papanastasiou A, Morsi-Yeroyannis A, Karagiannidis E, Kartas A, Doundoulakis I, Karvounis H and Giannakoulas G: Association of anticoagulant-related bleeding events with cancer detection in atrial fibrillation: A systematic review and meta-analysis. Hellenic J Cardiol 62: 359-365, 2021

33. Chu G, Versteeg HH, Verschoor AJ, Trines SA, Hemels ME, Ay C, Huisman MV and Klok FA: Atrial fibrillation and cancer-An unexplored field in cardiovascular oncology. Blood Rev 35: 59-67, 2019.

34. Keramida K, Filippatos G and Farmakis D: Cancer treatment and atrial fibrillation: Use of pharmacovigilance databases to detect cardiotoxicity. Eur Heart J Cardiovasc Pharmacother 7: 321-323, 2021.

35. Alexandre J, Salem JE, Moslehi J, Sassier M, Ropert C, Cautela J, Thuny F, Ederhy S, Cohen A, Damaj G, et al: Identification of anticancer drugs associated with atrial fibrillation: Analysis of the WHO pharmacovigilance database. Eur Heart J Cardiovase Pharmacother 7: 312-320, 2021.

36. Alexandre J, Moslehi JJ, Bersell KR, Funck-Brentano C, Roden DM and Salem JE: Anticancer drug-induced cardiac rhythm disorders: Current knowledge and basic underlying mechanisms. Pharmacol Ther 189: 89-103, 2018

37. Watson T, Shantsila E and Lip GY: Mechanisms of thrombogenesis in atrial fibrillation: Virchow's triad revisited. Lancet 373: 155-166, 2009.

38. Sethi G, Shanmugam MK, Ramachandran L, Kumar AP and Tergaonkar V: Multifaceted link between cancer and inflammation. Biosci Rep 32: 1-15, 2012.

39. Piazza $G$ and Ridker PM: Is venous thromboembolism a chronic inflammatory disease? Clin Chemistry 61: 313-316, 2015.

40. Morrell CN, Hilt ZT, Pariser DN and Maurya P: PAD4 and von Willebrand Factor Link Inflammation and Thrombosis. Circ Res 125: 520-522, 2019

41. Rickles FR, Patierno S and Fernandez PM: Tissue factor, thrombin, and cancer. Chest 124 (Suppl 3): 58S-68S, 2003.

42. Mousa SA: Anticoagulants in thrombosis and cancer: The missing link. Semin Thromb Hemost 28: 45-52, 2002

43. Allavena P, Garlanda C, Borrello MG, Sica A and Mantovani A Pathways connecting inflammation and cancer. Curr Opin Genet Dev 18: 3-10, 2008
44. Farmakis D, Chrysohoou C, Giamouzis G, Giannakoulas G, Hamilos M, Naka K, Tzeis S, Xydonas S, Karavidas A and Parissis J: The management of atrial fibrillation in heart failure: An expert panel consensus. Heart Fail Rev 26: 1345-1358, 2021.

45. Leiva O, AbdelHameid D, Connors JM, Cannon CP and Bhatt DL: Common pathophysiology in cancer, atrial fibrillation, atherosclerosis, and thrombosis: JACC: CardioOncology State-of-the-Art review. JACC CardioOncol 3: 619-634, 2021.

46. Saghazadeh A, Hafizi S and Rezaei N: Inflammation in venous thromboembolism: Cause or consequence? Int Immunopharmacol 28: 655-665, 2015.

47. Bikdeli B, Abou Ziki MD and Lip GYH: Pulmonary embolism and atrial fibrillation: Two sides of the same Coin? A systematic review. Semin Thromb Hemost 43: 849-863, 2017.

48. Melloni C, Shrader P, Carver J, Piccini JP, Thomas L, Fonarow GC, Ansell J, Gersh B, Go AS, Hylek E, et al: Management and outcomes of patients with atrial fibrillation and a history of cancer: the ORBIT-AF registry. Eur Heart J Qual Care Clin Outcomes 3: 192-197, 2017.

49. Pastori D, Marang A, Bisson A, Menichelli D, Herbert J, Lip GYH and Fauchier L: Thromboembolism, mortality, and bleeding in 2,435,541 atrial fibrillation patients with and without cancer: A nationwide cohort study. Cancer 127: 2122-2129, 2021

50. Kraaijpoel $\mathrm{N}$ and Carrier M: How I treat cancer-associated venous thromboembolism. Blood 133: 291-298, 2019.

51. Min JS and Bae SK: Prediction of drug-drug interaction potential using physiologically based pharmacokinetic modeling. Arch Pharm Res 40: 1356-1379, 2017.

52. van Leeuwen RW, Brundel DH, Neef C, van Gelder T, Mathijssen RH, Burger DM and Jansman FG: Prevalence of potential drug-drug interactions in cancer patients treated with oral anticancer drugs. Br J Cancer 108: 1071-1078, 2013.

53. Lechat P, Bergmann JF, Hanon PO, Beauvais F, Pol S, Cavagna F, Sicard J, Janus N, Deray G and Launay-Vacher V: Etude IPOP: Analyse qualitative et quantitative sur le risque iatrogénique chez les sujets âgés en France, en médecine de ville. La Lettre du Pharmacologue 30: 76-82, 2016 (In French)

54. Lee JY, Oh IY, Lee JH, Kim SY, Kwon SS, Yang HJ, Kim YK and Bang SM: The increased risk of bleeding due to drug-drug interactions in patients administered direct oral anticoagulants. Thromb Res 195: 243-249, 2020.

55. Ebner M, Birschmann I, Peter A, Härtig F, Spencer C, Kuhn J, Rupp A, Blumenstock G, Zuern CS, Ziemann U and Poli S: Limitations of specific coagulation tests for direct oral anticoagulants: A critical analysis. J Am Heart Assoc 7: e009807, 2018.

56. Boriani G,Lee G,Parrini I,Lopez-Fernandez T,Lyon AR, Suter T, Van der Meer P, Cardinale D, Lancellotti P, Zamorano JL, et al: Anticoagulation in patients with atrial fibrillation and active cancer: An international survey on patient management. Eur J Prev Cardiol 28: 611-621, 2021.

57. Russo AM: Anticoagulation in cancer patients with atrial fibrillation or atrial flutter: Are there gaps in care? JACC CardioOncol 2: 755-757, 2020.

58. Pangilinan JM, Pangilinan PH Jr and Worden FP: Use of warfarin in the patient with cancer. J Support Oncol 5: 131-136, 2007.

59. Ambrus DB, Reisman JI and Rose AJ: The impact of new-onset cancer among veterans who are receiving warfarin for atrial fibrillation and venous thromboembolism. Thromb Res 144: 21-26, 2016

60. Lee YJ, Park JK, Uhm JS, Kim JY, Pak HN, Lee MH, Sung JH and Joung B: Bleeding risk and major adverse events in patients with cancer on oral anticoagulation therapy. Int J Cardiol 203: 372-378, 2016.

61. Delluc A, Wang TF, Yap ES, Ay C, Schaefer J, Carrier M and Noble S: Anticoagulation of cancer patients with non-valvular atrial fibrillation receiving chemotherapy: Guidance from the SSC of the ISTH. J Thromb Haemost 17: 1247-1252, 2019.

62. Lee AY, Levine MN, Baker RI, Bowden C, Kakkar AK, Prins M, Rickles FR, Julian JA, Haley S, Kovacs MJ, et al: Low-molecular-weight heparin versus a coumarin for the prevention of recurrent venous thromboembolism in patients with cancer. N Engl J Med 349: 146-153, 2003.

63. Lee AYY, Kamphuisen PW, Meyer G, Bauersachs R, Janas MS, Jarner MF and Khorana AA; CATCH Investigators: Tinzaparin vs warfarin for treatment of acute venous thromboembolism in patients with active cancer: A randomized clinical trial. JAMA 314: 677-686, 2015.

64. Zhang N, Lou W, Ji F, Qiu L, Tsang BK and Di W: Low molecular weight heparin and cancer survival: clinical trials and experimental mechanisms. J Cancer Res Clin Oncol 142: 1807-1816, 2016 
65. Pfankuchen DB, Stolting DP, Schlesinger M, Royer HD and Bendas G: Low molecular weight heparin tinzaparin antagonizes cisplatin resistance of ovarian cancer cells. Biochem Pharmacol 97: 147-157, 2015.

66. Bokas A, Papakotoulas P, Sarantis P, Papadimitropoulou A, Papavassiliou AG and Karamouzis MV: Mechanisms of the antitumor activity of low molecular weight heparins in pancreatic adenocarcinomas. Cancers (Basel) 12: 432, 2020.

67. Poterucha TJ, Libby P and Goldhaber SZ: More than an anticoagulant: Do heparins have direct anti-inflammatory effects? Thromb Haemost 117: 437-444, 2017.

68. Klerk CP, Smorenburg SM, Otten HM, Lensing AW, Prins MH, Piovella F, Prandoni P, Bos MM, Richel DJ, van Tienhoven G and Büller HR: The effect of low molecular weight heparin on survival in patients with advanced malignancy. J Clin Oncol 23: 2130-2135, 2005

69. Calvo Romero JM: Low-molecular-weight heparins in patients with atrial fibrillation. Rev Clini Esp (Barc) 217: 151-154, 2017 (In English, Spanish).

70. Steinberg BA, Peterson ED, Kim S, Thomas L, Gersh BJ, Fonarow GC, Kowey PR, Mahaffey KW, Sherwood MW, Chang $\mathrm{P}$, et al: Use and outcomes associated with bridging during anticoagulation interruptions in patients with atrial fibrillation: Findings from the Outcomes Registry for Better Informed Treatment of Atrial Fibrillation (ORBIT-AF). Circulation 131: 488-494, 2015

71. Stellbrink C: Quick, easy, and safe? On the use of low-molecular-weight heparins in cardioversion of atrial fibrillation. Eur Heart J 27: 2742-2743, 2006.

72. Noble S, Matzdorff A, Maraveyas A, Holm MV and Pisa G: Assessing patients' anticoagulation preferences for the treatment of cancer-associated thrombosis using conjoint methodology. Haematologica 100: 1486-1492, 2015.

73. Konstantinides SV, Meyer G, Becattini C, Bueno H, Geersing GJ, Harjola VP, Huisman MV, Humbert M, Jennings CS Jiménez D, et al: 2019 ESC Guidelines for the diagnosis and management of acute pulmonary embolism developed in collaboration with the European Respiratory Society (ERS). Eur Heart J 41: 543-603, 2020

74. Agnelli G, Becattini C, Meyer G, Muñoz A, Huisman MV, Connors JM, Cohen A, Bauersachs R, Brenner B, Torbicki A, et al: Apixaban for the treatment of venous thromboembolism associated with cancer. N Engl J Med382: 1599-1607, 2020.

75. Sabatino J, Rosa SD, Polimeni A, Sorrentino S and Indolfi C: Direct Oral anticoagulants in patients with active cancer: A systematic review and Meta-analysis. JACC CardioOncol 2 : 428-440, 2020.

76. Key NS, Khorana AA, Kuderer NM, Bohlke K, Lee AYY, Arcelus JI, Wong SL, Balaban EP, Flowers CR, Francis CW, et al: Venous thromboembolism prophylaxis and treatment in patients with cancer: ASCO Clinical practice guideline update. J Clin Oncol 38: 496-520, 2020

77. Raskob GE, van Es N, Verhamme P, Carrier M, Di Nisio M, Garcia D, Grosso MA, Kakkar AK, Kovacs MJ, Mercuri MF, et al: Edoxaban for the treatment of cancer-associated venous thromboembolism. N Engl J Med 378: 615-624, 2018.

78. Chen ST, Hellkamp AS, Becker RC, Berkowitz SD, Breithardt G, Fox KAA, Hacke W, Halperin JL, Hankey GJ, Mahaffey KW, et al: Efficacy and safety of rivaroxaban vs. warfarin in patients with non-valvular atrial fibrillation and a history of cancer: Observations from ROCKET AF. Eur Heart J Qual Care Clini Outcomes 5: 145-152, 2019.

79. Melloni C, Dunning A, Granger CB, Thomas L, Khouri MG, Garcia DA, Hylek EM, Hanna M, Wallentin L, Gersh BJ, et al: Efficacy and safety of apixaban versus warfarin in patients with atrial fibrillation and a history of cancer: Insights from the ARISTOTLE trial. Am J Med 130: 1440-1448.e1, 2017.

80. Fanola CL, Ruff CT, Murphy SA, Jin J, Duggal A, Babilonia NA, Sritara P, Mercuri MF, Kamphuisen PW, Antman EM, et al: Efficacy and safety of edoxaban in patients with active malignancy and atrial fibrillation: Analysis of the ENGAGE AF-TIMI 48 trial. J Am Heart Assoc 7: e008987, 2018.

81. Shah S, Norby FL, Datta YH, Lutsey PL, MacLehose RF, Chen LY and Alonso A: Comparative effectiveness of direct oral anticoagulants and warfarin in patients with cancer and atrial fibrillation. Blood Adv 2: 200-209, 2018.
82. Sawant AC, Kumar A, Mccray W, Tetewsky S, Parone L, Sridhara S, Prakash MPH, Tse G, Liu T, Kanwar N, et al: Superior safety of direct oral anticoagulants compared to Warfarin in patients with atrial fibrillation and underlying cancer: A national veterans affairs database study. J Geriatr Cardiol 6: 706-709, 2019.

83. Mariani MV, Magnocavallo M, Straito M, Piro A, Severino P, Iannucci G, Chimenti C, Mancone M, Rocca DGD, Forleo GB, et al: Direct oral anticoagulants versus vitamin K antagonists in patients with atrial fibrillation and cancer a meta-analysis. J Thromb Thrombolysis 51: 419-429, 2021.

84. Deng Y, Tong Y, Deng Y,Zou L, Li S and Chen H: Non-Vitamin K antagonist oral anticoagulants versus warfarin in patients with cancer and atrial fibrillation: A systematic review and meta-analysis. J the Am Heart Assoc 8: e012540, 2019.

85. Steffel J, Verhamme P, Potpara TS, Albaladejo P, Antz M, Desteghe L, Haeusler KG, Oldgren J, Reinecke H, Roldan-Schilling V, et al: The 2018 European Heart Rhythm association practical guide on the use of non-vitamin $\mathrm{K}$ antagonist oral anticoagulants in patients with atrial fibrillation. Eur Heart J 39: 1330-1393, 2018.

86. Tarn DM, Barrientos M, Wang AY, Ramaprasad A, Fang MC and Schwartz JB: Prevalence and knowledge of potential interactions between over-the-counter products and apixaban. J Am Geriat Soc 68: 155-162, 2020

87. Lopez-Fernandez T,Martin-Garcia A,Roldan Rabadan I,MitroiC, Mazón Ramos P, Díez-Villanueva P, Escobar Cervantes C, Alonso Martín C, Alonso Salinas GL, Arenas M, et al: Atrial fibrillation in active cancer patients: Expert position paper and recommendations. Rev Esp Cardiol (Engl Ed) 72: 749-759, 2019 (in English, Spanish).

88. Zamorano JL, Lancellotti P, Rodriguez Muñoz D, Aboyans V, Asteggiano R, Galderisi M, Habib G, Lenihan DJ, Lip GYH, Lyon AR, et al: 2016 ESC Position Paper on cancer treatments and cardiovascular toxicity developed under the auspices of the ESC Committee for Practice Guidelines: The Task Force for cancer treatments and cardiovascular toxicity of the European Society of Cardiology (ESC). Eur Heart J 37: 2768-2801, 2016.

89. Fradley MG, Ellenberg K, Alomar M, Swanson J, Kharod A Nguyen ATH, Khodor S, Mishra S, Duong LM, Shah N, et al: Patterns of anticoagulation use in patients with cancer with atrial fibrillation and/or atrial flutter. JACC CardioOncol 2: 747-754, 2020.

90. Malavasi VL, Fantecchi E, Gianolio L, Pesce F, Longo G, Marietta M, Cascinu S, Lip GYH and Boriani G: Atrial fibrillation in patients with active malignancy and use of anticoagulants: Under-prescription but no adverse impact on all-cause mortality. Eur J Intern Med 59: 27-33, 2019.

91. Farmakis D: Is cardio-oncology a rapidly growing field of precision medicine? Eur J Heart Fail 22: 2310-2313, 2020.

92. Farmakis D, Keramida K and Filippatos G: How to build a cardio-oncology service? Eur J Heart Fail 20: 1732-1734, 2018.

93. Mbroh J and Poli S: 2021 European Heart Rhythm Association Practical Guide on the use of non-vitamin K antagonist oral anticoagulants in patients with atrial fibrillation: comment. Europace 23: 1685, 2021.

94. Miller KD, Nogueira L, Mariotto AB, Rowland JH, Yabroff KR, Alfano CM, Jemal A, Kramer JL and Siegel RL: Cancer treatment and survivorship statistics, 2019. CA Cancer J Clin 69: 363-385, 2019.

95. Zaorsky NG, Churilla TM, Egleston BL, Fisher SG, Ridge JA, Horwitz EM and Meyer JE: Causes of death among cancer patients. Ann Oncol 28: 400-407, 2017.

96. Russo AM: Anticoagulation in cancer patients with atrial fibrillation or atrial flutter. JACC CardioOncol 2: 755-757, 2020.

97. Piccini JP, Xu H, Cox M, Matsouaka RA, Fonarow GC, Butler J, Curtis AB, Desai N, Fang M, McCabe PJ, et al: Adherence to guideline-directed stroke prevention therapy for atrial fibrillation is achievable. Circulation 139: 1497-1506, 2019.

This work is licensed under a Creative Commons Attribution-NonCommercial-NoDerivatives 4.0 International (CC BY-NC-ND 4.0) License. 